\title{
Detecting Infarct Region in Cardiac Magnetic Resonance Images Through Weighted Normalized Mutual Information
}

\author{
Hossein Yousefi-Banaem, ${ }^{1}$ Saeed Kermani, ${ }^{1,}{ }^{*}$ Hamid Sanei, ${ }^{2}$ and Alireza Daneshmehr ${ }^{3}$ \\ ${ }^{1}$ Department of Biomedical Engineering, Isfahan University of Medical Sciences, Isfahan, Iran \\ ${ }^{2}$ Department of Cardiology, School of Medicine, Isfahan University of Medical Sciences, Isfahan, Iran \\ ${ }^{3}$ Department of Mechanical Engineering, School of Engineering, University of Tehran, Tehran, Iran \\ "Corresponding author: Saeed Kermani, Department of Biomedical Engineering Faculty of Advance Medical Technology, Isfahan University of Medical Sciences, Isfahan, Iran. \\ Tel: +98-9133107346, E-mail: kermani@med.mui.ac.ir.
}

Received 2016 August 08; Revised 2016 August 13; Accepted 2016 September 04.

\begin{abstract}
Background: Myocardial infarction remains a leading cause of morbidity and mortality among cardiac disease. Cardiac wall thickening in patients with myocardial infarction is less than healthy individuals. Accurate measurement of cardiac wall fractional thickening and path-length of myocardium points in healthy data and patients with myocardial infarction can help physicians in diagnosing the affected area.

Patients and Methods: Epi/Endocardium of all slices in end-diastole frame were segmented, then more than 150 points in each slice were selected to track by weighted normalized mutual information algorithm over all frames. Weighted normalized mutual information was computed between two three-dimensional masks sized $3 \times 3 \times 3$, pixel that were located in end-diastole and subsequent frames centroid of the selected points. Finally, by computing the distance between endocardium and epicardium in each slice over all frames, cardiac wall thickness and fractional thickening was measured. Moreover, the path-length of each data point during cardiac period was calculated and sketched in bulls-eye format. Evaluation of the method was done by ten healthy and twenty patients with myocardial infarction.

Results: Cardiac wall kinesis was evaluated by normalized path length, which was presented in standard 17-segment bull's-eye format. Wall thickness and fractional wall thickening for all slices over all frames were extracted in order to determine the infarct region. Infarct regions had minimal fractional thickening and normalized path length. All evaluations demonstrated hypo-kinesis in the damaged region.

Conclusion: Evaluation of obtained results showed significant difference between local parameters of healthy and infarcted myocardium. In all patients, the process was able to precisely determine the affected region that was all well matched with clinical evidence.
\end{abstract}

Keywords: Myocardium, Infarction, Normalized Mutual Information, Motion Tracking

\section{Background}

Acute myocardial infarction (AMI) is the main cause of death throughout the world despite diagnostic developments and therapeutic improvements (1). Patients with AMI have high mortality. Local left ventricular function is altered during and after AMI. Most studies describe changes in the infarcted myocardium. These changes occur in the infarcted, ischemic and adjacent regions of the myocardium $(2,3)$. There is less information about changes in the remote region of AMI in the myocardium. It is still argumentative whether remote myocardium after AMI is hypo-functioning or hyper functioning (4-6). This has not been well studied by cardiac imaging.

Cardiac magnetic resonance imaging (CMRI) is a powerful diagnostic tool that can provide more accurate measurements of cardiac chambers, volumes, dimensions (7), local cardiac wall function (8), and infarct area extent (9).
It has been considered as the gold standard for evaluating systolic wall thickening (6). Studies have shown that regional wall function can be assessed using CMR strain analysis (10). Strain is a measure of the change in size and shape of an object and can be derived from CMR by using grid-tagging (11), displacement encoding with stimulated echoes (DENSE) (12) or velocity-encoded (VE) imaging (13). Studies conducted by Kroeker et al. and Garot et al. found that myocardial infarction decrease left ventricular (LV) thickness and twist (rotation of heart muscle during contraction) during LV contraction $(14,15)$. Therefore, to obtain the corresponding point in the next frame and to assess function of the myocardium, point tracking methods have been used.

Heart wall motion tracking methods can be divided into two groups: local point tracking and image registration. In local point tracking, the first selected points are tracked from diastole to systole. Subsequently, dense mo- 
tion field is estimated. Reconstruction dense motion field from a sparse set of control points is an ill-posed problem. Therefore, to have a unique solution, we need additional constraints. These limitations have previously been suggested in the literature (16). The other method for motion tracking is whole image registration or feature point registration (17). Dinan et al. used block matching to track the end diastole selected points over all frames (18). Kermani et al. proposed correlation based 3D block matching to track sparse point during cardiac cycle (19). They used Sobel based gradient operator that works on edges. Gradient based methods do not contain information about the region, so they may cause miss tracking. Therefore, a method that carries information from the region is required. Some methods such as mutual information (MI) and normalized mutual information (NMI) could give useful information from the determined region in order to compute the similarities by extracting some nonlinear feathers (20-23). NMI is a powerful method to measure the similarities of two images $(8,20,24)$. Tahan et al. used NMI as a similarity metric in the registration of two sequential cardiac tagged MRIs. In tagged MRI, tag lines vanish during the cardiac contraction cycle (25). Zhang et al. applied NMI as a similarity metric in order to track coronary artery motion based on non-rigid registration method in $4 \mathrm{D}$ cardiac $\mathrm{CT}$ angiogram data sets $(24,26)$. Beache et al. used NMI in cardiac perfusion data to evaluate myocardium functionality by affinebased registration. They demonstrated that this method could assess the cardiac wall properly (27). Most of these methods have used MI or NMI in order to measure whole image similarities. In the present study, similarities were calculates in small sub images, so subtle alteration in density and shape caused considerable alteration in similarity measurements. Another similarity metric tool is correlation coefficient, which is only able to take into account linear relationships. Correlation coefficient is a good measure in a case of similar subject and modality (28). Because of using the same modalities in the tracking method, we proposed correlation coefficient based weighted-NMI, in order to improve the result accuracy by considering both linear and nonlinear features. Therefore, this study was constructed to assess local myocardial wall function by measuring path-length and fractional wall thickening by tracking some points in the LV wall from end-diastole to end-systole using weighted-NMI. Obtained features of acute myocardial infarct patients compared with healthy individuals by quantizing wall motion.

This paper is organized as follows: first CMRI images were segmented over all slices in end-diastole frame, and then selected boundary points were tracked by weightedNMI to extract the local dynamic and functional LV parameters. Then measured fractional thickening and path-length of healthy and infarct regions were visualized. We have also validated our method with real data acquired from patients and the infarct regions were successfully located with outstanding reliability and accuracy.

\section{Patients and Methods}

An overview of the proposed method is demonstrated in Figure 1.

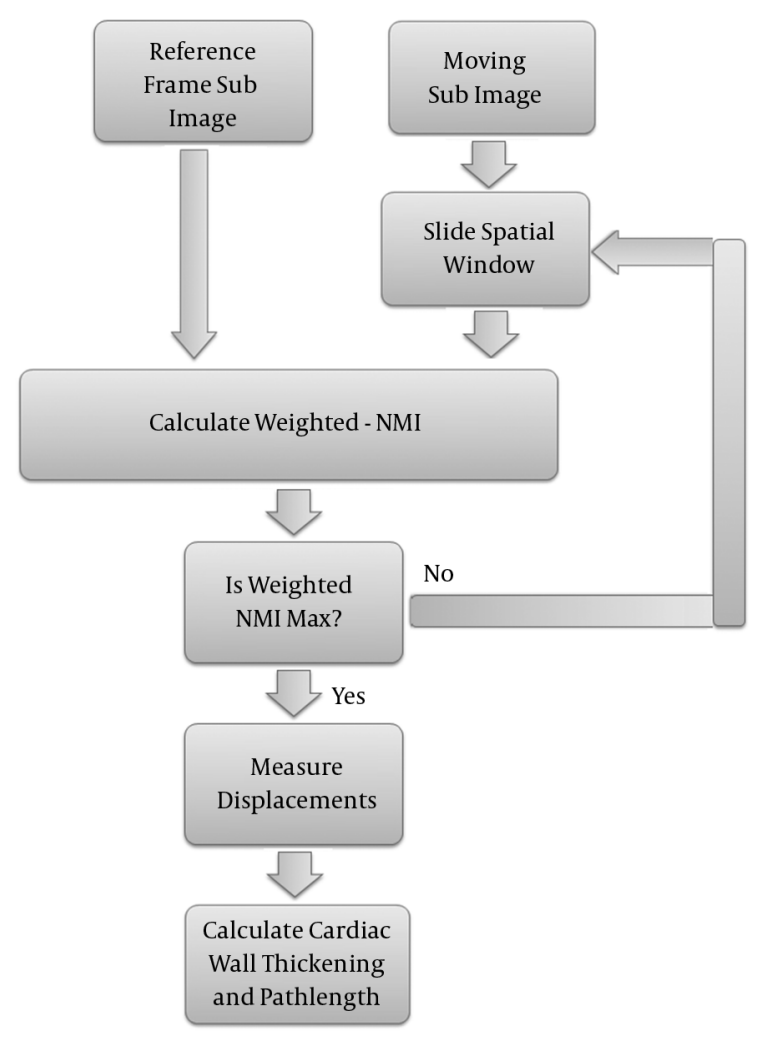

Figure 1. Block diagram of the proposed method (NMI; normalized mutual information)

\subsection{CMRI Imaging}

Real patient geometric data with myocardial infarct was selected from three cine MRI databases. Eleven gradient-echo images were performed on a $1.5 \mathrm{~T}$ scanner (Symphony Siemens, Germany) in Isfahan MRI Center, Isfahan, Iran (19). Twenty of the images were obtained from Sunnybrook health sciences centre, Toronto, Canada (29) and 20 of them were obtained from cardiac atlas project (30). The implemented MATLAB program runs in a computer with dual core $2.53 \mathrm{GHz}$ CPU and 16G of RAM. Information of patient has brought in Table 1. 


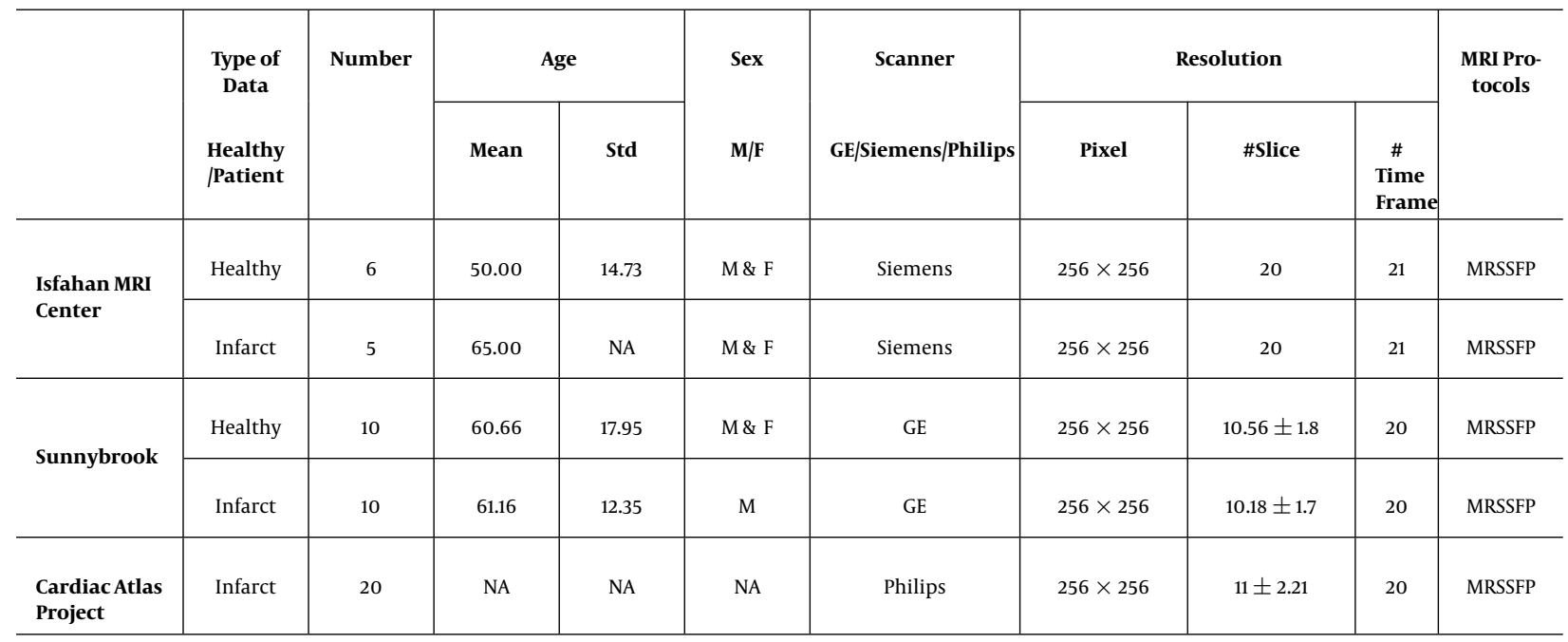

${ }^{a}$ For assessment of the local cardiac wall, steady state free precession (SSFP) cine MRI images were acquired in the short-axis plane covering the entire left ventricle from base to apex. Image parameters were: repetition time $3.2 \mathrm{~ms}$, echo time $1.6 \mathrm{~ms}$, flip angle $60^{\circ}$, image resolution $1.36 \times 1.36 \mathrm{~mm}$, slice thickness $8 \mathrm{~mm}$, retrospective ECG gated reconstruction.

\subsection{Myocardium Segmentation}

Epi/Endocardial of all slices in all frames, are segmented exactly based on the algorithm introduced by Heiberg et al. (31). This method is developed on the concept of deformable models but extended with an enhanced and fast edge detection scheme that includes temporal information and anatomical priori knowledge. In this method, LV model is a time-resolved mesh representation of the LV as an open cone, sliced along the cone's long axis with 80 numbers of points in each slice. Results of this algorithm are not accurate among all slices and frames, so all segmentations were refined by an expert until contours coincided to the epi/endocardium.

\subsection{Sparse Point Tracking}

To obtain the sparse field of displacement, the point on the epi/endocardial surfaces were used. To track the point displacement and determination of the initial sparse motion field, efficient weighted-NMI algorithm was used. This approach was implemented on two sequential frames of a 3D sequence image. First, 3D reference mask with center of selected points in end diastole frame was constructed. Then, a moving mask with the same size as the generated mask with a close center to the reference mask was constructed in the next frame. The moving mask moves in all directions and weighted-NMI of the moving mask and reference mask was calculated. Then, the center points of the two masks with high weighted-NMI were considered as the corresponding points. The center point of the moving mask with high weighted-NMI was considered as the reference mask, and the moving mask is transferred to the next frame. This procedure continued until the moving mask reached the end systole frame. Finally, the tracking process was repeated for all selected points in end diastole frame to measure their displacements during the cardiac cycle.

\subsection{Weighted-NMI}

One way to measure the similarities of two images is MI. It uses joint entropy and marginal entropy to calculate the common information between the two images based on Equation 1:

$M I(X, Y)=H(X)+H(Y)-H(X, Y)$

Where $\mathrm{H}(\mathrm{X})$ and $\mathrm{H}(\mathrm{Y})$ are marginal entropy, and $\mathrm{H}(\mathrm{X}, \mathrm{Y})$ is joint (Shannon) entropy, which is obtained as Equations $2-4$.

$H(X, Y)=-\sum_{x \epsilon X} \sum_{y \in Y} P x y(X, Y) \log P x y(X, Y)$

$H_{x}=\sum_{x \in X} P x(X) \log P x(X)$

$H_{y}=\sum_{y \in Y} p_{y}(y) \log p_{y}(y)$

Where $\mathrm{P}_{\mathrm{x}}(\mathrm{x}), \mathrm{P}_{\mathrm{y}}(\mathrm{y})$, and $\mathrm{P}_{\mathrm{xy}}(\mathrm{x}, \mathrm{y})$ are marginal probability density function and joint probability density function, respectively and obtained as Equations 5 and 6. 
$p_{x}(x)=\sum_{y} p_{x y}(x, y)$

$p_{y}(y)=\sum_{x} p_{x y}(x, y)$

In MI, joint entropy and marginal entropy of the moving image vary with changing overlap, so it is not appropriate for tracking a point. In order to provide overlap invariance, we need a measure independent of changes in the marginal entropies $\mathrm{H}(\mathrm{X})$ and $\mathrm{H}(\mathrm{Y})$ of the two images in their region of overlap. A direct approach is normalization, which was used in this paper to evaluate the ratio of the joint and marginal entropies. On the other hand, since correlation coefficient is a better measure of similarity in the case of similar subject and modality, it is used to detect changes in intensity or the shape of a structure. Correlation coefficient metric was computed pixel-wise and misalignment between the images resulted in small measure values. We used correlation coefficient to weight the NMI as Equation 7. Weighted-NMI needs both linear and nonlinear properties of two compared images so the accuracy of tracking can be improved.

Weighted $-N M I(X, Y)=C C \times \frac{H(X)+H(Y)}{H(X, Y)}$

Where CC is the correlation coefficient as a weight (Equation 8).

$C C=\frac{\left(N \sum I_{1} I_{2}\right)-\left(\sum I_{1}\right)\left(\sum I_{2}\right)}{\sqrt{\left[N \sum I_{1}^{2}-\left(\sum I_{1}\right)^{2}\right]\left[N \sum I_{2}^{2}-\left(\sum I_{2}\right)^{2}\right]}}$

Where, $\mathrm{I}_{1}$ and $\mathrm{I}_{2}$ are the reference frame mask and moving mask in the adjacent frame, respectively, and $\mathrm{N}$ is the number of voxels.

Correspondent point in the next frame obtained by maximizing the weighted-NMI as Equation 9:

$C P=\arg \quad \max \quad$ weighted $-N M I(X, Y)$

Finally, by subtracting two points - reference point and its correspondent point in the next frame - the displacements were obtained as Equation 10:

Displacement $=P_{R}-C P_{N F}$

Where $\mathrm{P}_{\mathrm{R}}$ stands for reference points, and $\mathrm{CP}_{\mathrm{NF}}$ stands for the correspondent point in the next frame. After calculating the displacements from systole to diastole, useful parameters for infarct region detection are extracted.

\subsection{Wall Thickening}

For assessment of local wall thickening from the shortaxis cine MRI images, cardiac wall thickness, wall thickening, and fractional wall thickening were computed after manual refining of the endo/epicardial borders. The left ventricle was divided into eight sectors in each slice. For result improvement, papillary muscles were excluded from the myocardium segmentation. Basal slice in short axis is the first slice of cardiac cine MRI that contains all LV wall in the end diastole frame. To measure LV wall thickness, Euclidian distance between endocardium and epicardium points is calculated as Equation 11:

$W T(x, y)=\sqrt{\left(x_{\text {endo }}-x_{\text {epi }}\right)^{2}+\left(y_{\text {endo }}-y_{\text {epi }}\right)^{2}}$

In a healthy heart during ventricular contraction, the LV wall begins to thicken. LV wall thickening rate is defined as difference between LV wall thicknesses in two successive frames (Equation 12):

$w t=W T_{f+1}-W T_{f} ; f=1 ;$ Number offrame

Since LV wall thickness differs in different direction of slices, LV wall thickening is normalized. Fractional wall thickening defined as wall thickening is divided by end diastolic wall thickness as Equation 13:

$f w t=\frac{w t}{W T_{\text {end-diastole }}}$

\subsection{Path Length Measurement}

Path length is defined as the total distance a points moves. Unlike displacement, which is the total distance a point moves from the end diastole frame to the end systole frame, path length is the total distance travelled, regardless of where it moved (Equation 14).

$l=\sum_{i, j} D_{i, j}$

$D_{j i}$ is the distance between nodes in frames $i$ and $j$. Polar map representation of path length for patients has been presented in 17-segment bulls-eye.

\section{Results}

As illustrated above for myocardium functionality evaluation we measured several local parameters including cardiac wall thickening, fractional thickening and path-length of boundary points. In the first step, cardiac wall in the end diastole frame was segmented then boundary points were tracked using weighted-NMI. Segmentation results and trajectory of boundary points are demonstrated in Figure 2. 
A

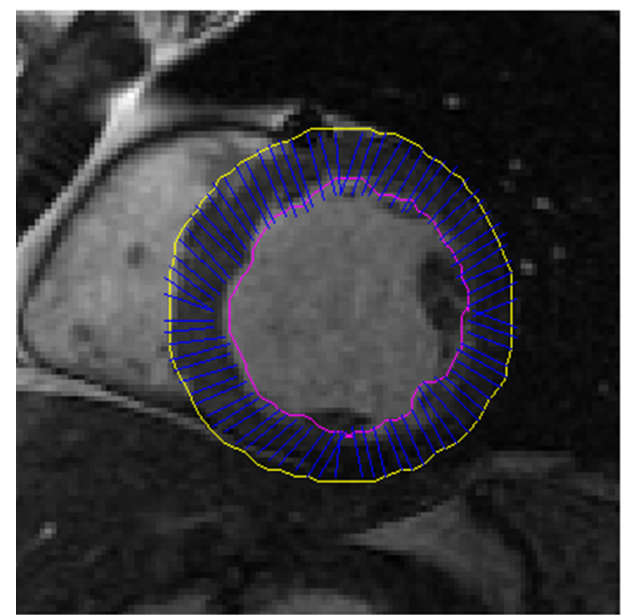

B

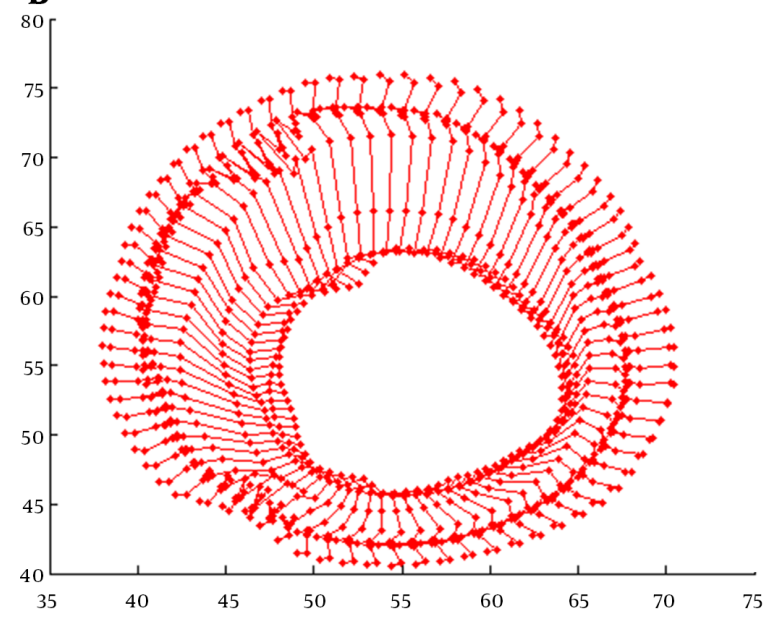

Figure 2. A, Segmented myocardium with blue lines that indicate wall thickness. B, Trajectory of endocardium from end-diastole to end systole

Since this method generates 3-D path-length fields for all 3-D points, for better evaluation we divided the entire LV wall into three sections: the basal layer, the midventricular layer, and the apical layer. Furthermore, the apical layer was divided into four regions: anterior, lateral, inferior, and septal. The mid-ventricular layer was divided into six regions: antero-septal, anterior, lateral, posterior, inferior, and infero-septal. Finally, the basal layer was divided into antero-septal, anterior, lateral, posterior, inferior, and infero-septal regions. The normalized pathlengths of LV wall over each of the 16 segmented regions were plotted in standard bull's-eye format. Normalized path-length of LV wall in standard bull's eye format is demonstrated in Figure 3.

Cardiac wall fractional thickening was evaluated as a measure of myocardium performance. Fractional thickening was calculated for all data points over time (cardiac cycle time). Cardiac wall in short axis was considered as a circle and divided into eight sectors as shown in Figure 4, then fractional thickening of the cardiac wall was computed in each sector.

Because the fractional thickening was computed over all frames, we plotted it as a function of time. For all plots, end-diastole was considered as the reference frame for calculating fractional thickening. The magnitude of fractional thickening values generally increases as the left ventricle contracts during systole. In infarct regions, the magnitude of fractional thickening is less than normal regions as showed in Figures 5 and 6.

\section{Discussion}

Current study was performed to evaluate myocardial functionality using weighted normalized mutual information for cardiac wall boundary point tracking. Myocardial function was assessed by measuring normalized pathlength of boundary points and fractional wall thickening calculation. We investigated the problem of detecting and localizing myocardium abnormality. The 3D motion of LV was tracked throughout the entire cardiac cycle, and a quantitative analysis was performed. The results of fractional wall thickening showed that wall thickness increases from diastole to systole phase of the cardiac cycle. In patients with infarction, wall thickening in the infarct region of the myocardium is less than the healthy region as illustrated in figures 5 and 6 . This finding is well matched with clinical findings caused when an infarction occurs; the myocardial muscle loses its elasticity and function. The infarct region of the myocardial muscle does not contract. Subsequently, the wall thickness is less subject to change. For better visualization and interpretation, results of normalized path-length have been sketched in 17 bull's-eye format, which maps 3D cardiac wall to 2D circle. As mentioned before, each segment in bull's-eye belongs to a specific region of the heart. So by plotting normalized path-length in bull'-eye format, the infarct region could be determined. In addition, the extent and severity of the infarction could be determined. As shown in figure 3, one patient has infarction on all sides of the wall and the other patient has extensive infarction from basal to mid ventricular LV but with more severity in the basal region, which is consistent with clinical findings. Unfortunately, the researchers failed to 

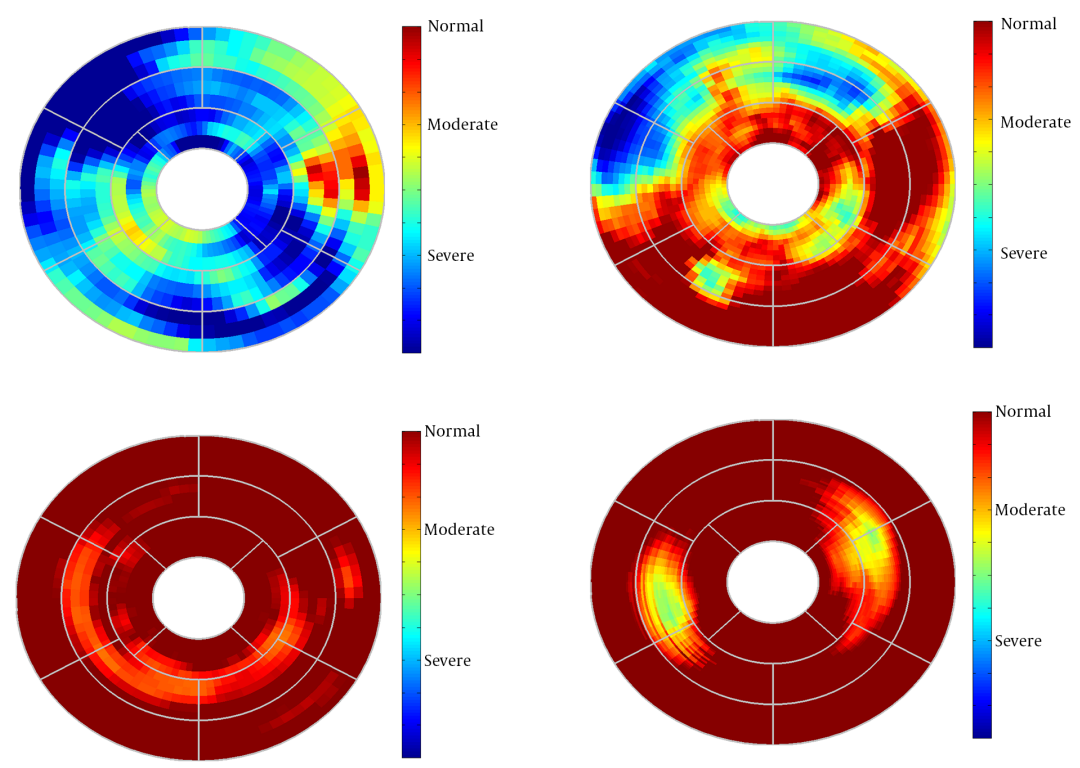

Figure 3. Standard bull's-eye of normalized path-length of cardiac wall boundary points. Dark red color indicates the healthy region of the cardiac wall with normal pathlength and dark blue color shows the infarct region with a less normalized path-length.

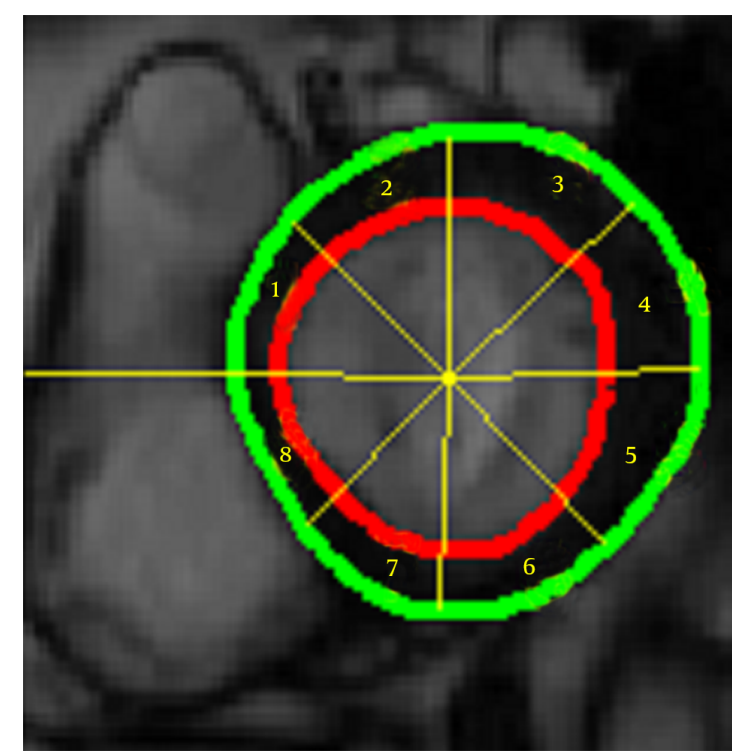

Figure 4. Cardiac wall was divided into sectors to measure the fractional thickening.

get access to CMRI sequences with implanted markers for comparison between marker trajectories and trajectories which have been derived from the proposed algorithm.

In conclusion, our proposed method is capable of producing comprehensive myocardial fractional wall thicken- ing and path-length calculations for assessing myocardial functionality by measuring displacement fields based on tracking procedure. The novelty of the method is that at the conclusion of point tracking, cardiac wall boundary point displacements, myocardium boundary point pathlength and fractional thickenings of the myocardium are immediately available. We illustrated these with results from 51 real human cardiac MRIs. The resulting pathlength values were normalized across studies and were shown in standard bull'-eye format and the fractional wall thickening of the heart was plotted over the systolic phase of the cardiac cycle. Results demonstrated that this method could be used for fast evaluation of myocardium functionality. 


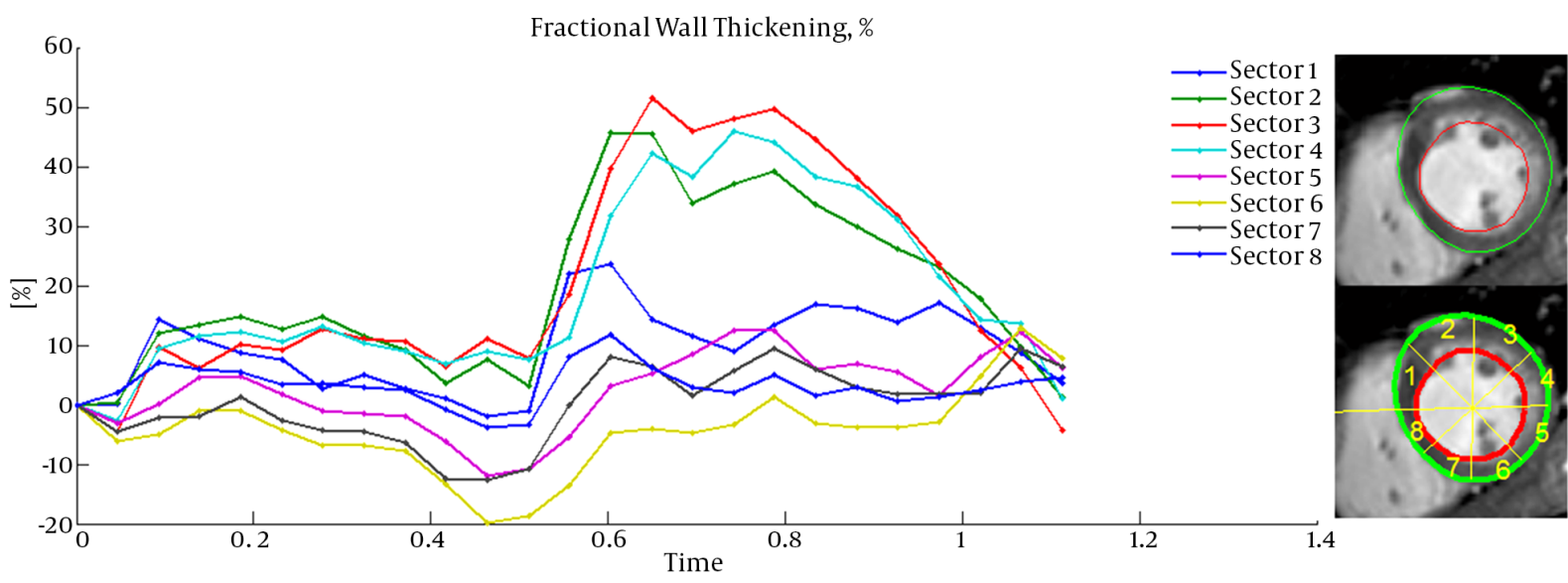

Figure 5. Fractional thickening of infarct myocardium. Infarct regions have less fractional thickening compared to normal regions.

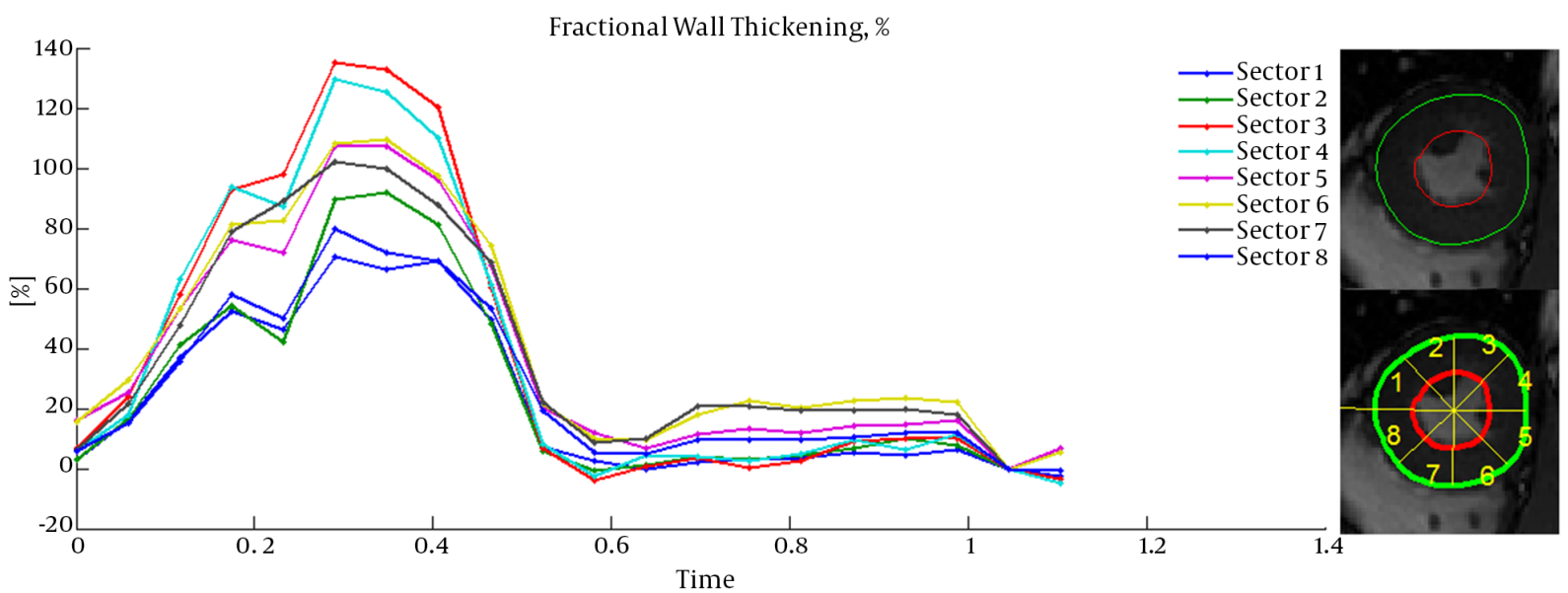

Figure 6. Fractional thickening of the healthy myocardium. All sectors have relatively high fractional thickening.

\section{Acknowledgments}

Authors would like to highly acknowledge the Cardiac Atlas Project funder for catering public cardiac MRI database, especially Avan Suinesiaputra for DETERMINE data access.

\section{Footnotes}

Authors' Contributions: Study concept and design: Hossein Yousefi-Banaem and Saeed Kermani; analysis and interpretation of data: Hossein Yousefi-Banaem; drafting of the manuscript: Hossein Yousefi-Banaem; revision of the manuscript for important intellectual content: Saeed Kermani and Hamid Sanei; clinical evaluation: Hamid Sanei

Financial Disclosure: None declared.

Funding/Support: This study was supported by Isfahan University of Medical Sciences.

\section{References}

1. Go A, Mozaffarian D, Roger V, Benjamin E, Berry J, Borden W. Heart disease and stroke statistics-2013 update: a report from the American Heart Association Circulation. American Heart Association statistics committee and stroke statistics subcommittee; 2013.

2. Rademakers F, Van de Werf F, Mortelmans L, Marchal G, Bogaert J. Evolution of regional performance after an acute anterior myocardial 
infarction in humans using magnetic resonance tagging. J Physiol. 2003;546(Pt 3):777-87. [PubMed: 12563003].

3. Shimkunas R, Zhang Z, Wenk JF, Soleimani M, Khazalpour M, AcevedoBolton G, et al. Left ventricular myocardial contractility is depressed in the borderzone after posterolateral myocardial infarction. Ann Thorac Surg. 2013;95(5):1619-25. doi: 10.1016/j.athoracsur.2013.02.005. [PubMed: 23523189].

4. Ito S, Suzuki T, Hosokawa H, Inada T, Takeda Y, Suzumura H, et al. Increased hyperkinesis in noninfarcted areas during short-term followup in patients with first anterior acute myocardial infarction treated by direct percutaneous transluminal coronary angioplasty.Jpn Heart J. 1999;40(5):549-60. [PubMed: 10888375].

5. Kramer CM, Rogers WJ, Theobald TM, Power TP, Petruolo S, Reichek N. Remote noninfarcted region dysfunction soon after first anterior myocardial infarction. A magnetic resonance tagging study. Circulation. 1996;94(4):660-6. [PubMed: 8772685].

6. Pahlm US, Ubachs JF, Heiberg E, Engblom H, Erlinge D, Gotberg $\mathrm{M}$, et al. Regional wall function before and after acute myocardial infarction; an experimental study in pigs. BMC Cardiovasc Disord. 2014;14:118. doi: 10.1186/1471-2261-14-118. [PubMed: 25218585].

7. Pennell DJ. Ventricular volume and mass by CMR. J Cardiovasc Magn Reson. 2002;4(4):507-13. [PubMed: 12549238].

8. Maceira AM, Prasad SK, Khan M, Pennell DJ. Normalized left ventricular systolic and diastolic function by steady state free precession cardiovascular magnetic resonance. J Cardiovasc Magn Reson. 2006;8(3):417-26. [PubMed: 16755827].

9. Pennell DJ, Sechtem UP, Higgins CB, Manning WJ, Pohost GM, Rademakers FE, et al. Clinical indications for cardiovascular magnetic resonance (CMR): Consensus Panel report. J Cardiovasc Magn Reson. 2004;6(4):727-65. [PubMed: 15646878].

10. Heiberg E, Pahlm-Webb U, Agarwal S, Bergvall E, Fransson H, StedingEhrenborg K, et al. Longitudinal strain from velocity encoded cardiovascular magnetic resonance: a validation study.J Cardiovasc Magn Reson. 2013;15:15. doi: 10.1186/1532-429X-15-15. [PubMed: 23343426].

11. Petitjean C, Rougon N, Cluzel P. Assessment of myocardial function: a review of quantification methods and results using tagged MRI. $J$ Cardiovasc Magn Reson. 2005;7(2):501-16. [PubMed: 15881535].

12. Zhong X, Helm PA, Epstein FH. Balanced multipoint displacement encoding for DENSE MRI. Magn Reson Med. 2009;61(4):981-8. doi: 10.1002/mrm.21851. [PubMed: 19189288].

13. Wedeen VJ. Magnetic resonance imaging of myocardial kinematics. Technique to detect, localize, and quantify the strain rates of the active human myocardium. Magn Reson Med. 1992;27(1):52-67. [PubMed: 1435210].

14. Kroeker CA, Tyberg JV, Beyar R. Effects of ischemia on left ventricular apex rotation. An experimental study in anesthetized dogs. Circulation. 1995;92(12):3539-48. [PubMed: 8521577].

15. Garot J, Derumeaux GA, Monin JL, Duval-Moulin AM, Simon M, Pascal D, et al. Quantitative systolic and diastolic transmyocardial velocity gradients assessed by M-mode colour Doppler tissue imaging as reliable indicators of regional left ventricular function after acute myocardial infarction. Eur Heart J. 1999;20(8):593-603. [PubMed: 10337544].

16. Montagnat J, Delingette H. 4D deformable models with temporal constraints: application to $4 \mathrm{D}$ cardiac image segmentation. Med Image Anal. 2005;9(1):87-100. doi: 10.1016/j.media.2004.06.025. [PubMed:
15581814].

17. Park J, Metaxas D, Axel L. Analysis of left ventricular wall motion based on volumetric deformable models and MRI-SPAMM. Med Image Anal. 1996;1(1):53-71. [PubMed: 9873921].

18. Dinan FJ, Mosayebi P, Moghadam HA, Giti M, Kermani S. A fully 3D system for cardiac wall deformation analysis in MRI data. Functional Imaging and Modeling of the Heart. Springer; 2007.

19. Kermani S, Moradi MH, Abrishami-Moghaddam H, Saneei H, Marashi MJ, Shahbazi-Gahrouei D. Quantitative analysis of left ventricular performance from sequences of cardiac magnetic resonance imaging using active mesh model. Comput Med Imaging Graph. 2009;33(3):22234. doi: 10.1016/j.compmedimag.2008.12.005. [PubMed: 19196492].

20. McDaid AF, Greene D, Hurley N. Normalized mutual information to evaluate overlapping community finding algorithms 2011. Available from: https://arxiv.org/abs/1110.2515.

21. Cahill ND. Normalized measures of mutual information with general definitions of entropy for multimodal image registration. Springer 2010.

22. Mukherjee JM, Hutton BF, Johnson KL, Pretorius PH, King MA. An evaluation of data-driven motion estimation in comparison to the usage of external-surrogates in cardiac SPECT imaging. Phys Med Biol. 2013;58(21):7625-46. doi: 10.1088/0031-9155/58/21/7625. [PubMed: 24107647].

23. Yousefi-Banaem H, Kermani S, Daneshmehr A, Saneie H. Application of Hyperelastic-based Active Mesh Model in Cardiac Motion Recovery. J Med Signals Sens. 2016;6(3):141-9. [PubMed: 27563570].

24. Zhang DP, Zhuang X, Ourselin S, Rueckert D. Motion tracking of left ventricle and coronaries in $4 \mathrm{D}$ CTA. SPIE Medical Imaging. International Society for Optics and Photonics; 2011.

25. Tahan S, Melli LC, Mello CS, Rodrigues MS, Bezerra Filho H, de Morais MB. Effectiveness of trimethoprim-sulfamethoxazole and metronidazole in the treatment of small intestinal bacterial overgrowth in children living in a slum.J Pediatr Gastroenterol Nutr. 2013;57(3):316-8. doi: 10.1097/MPG.ob013e3182952e93. [PubMed: 23974062].

26. Zhang DP, Risser L, Friman O, Metz C, Neefjes L, Mollet N, et al. Nonrigid registration and template matching for coronary motion modeling from 4D CTA. Springer; 2010.

27. Beache GM, Khalifa F, El-Baz A, Gimel'farb G. Fully automated framework for the analysis of myocardial first-pass perfusion MR images. Med Phys. 2014;41(10):102305. doi: 10.1118/1.4893531. [PubMed: 25281975].

28. Rueckert D, Schnabel JA. Medical image registration. Biomedical Image Processing. Springer; 2010.

29. Alfakih K, Plein S, Thiele H, Jones T, Ridgway JP, Sivananthan MU. Normal human left and right ventricular dimensions for MRI as assessed by turbo gradient echo and steady-state free precession imaging sequences. J Magn Reson Imaging. 2003;17(3):323-9. doi 10.1002/jmri.10262. [PubMed: 12594722].

30. Fonseca CG, Backhaus M, Bluemke DA, Britten RD, Chung JD, Cowan $\mathrm{BR}$, et al. The Cardiac Atlas Project-an imaging database for computational modeling and statistical atlases of the heart. Bioinformat ics. 2011;27(16):2288-95. doi: 10.1093/bioinformatics/btr360. [PubMed: 21737439].

31. Heiberg E, Wigstrom L, Carlsson M, Bolger A, Karlsson M. Time resolved three-dimensional automated segmentation of the left ventricle. Computers in Cardiology, 2005. IEEE; 2005. pp. 599-602. 